\title{
Emergence of dispersion in shallow water hydrodynamics via modulation of uniform flow
}

\author{
Thomas J. Bridges $\dagger$ \\ Department of Mathematics, University of Surrey, Guildford, Surrey GU2 7XH UK
}

(Received ?; revised ?; accepted ?)

A new theory for the emergence of dispersion in shallow-water hydrodynamics in two horizontal-space dimensions is presented. Starting with the key properties of uniform flow in open channel hydraulics, it is shown that criticality is the key mechanism for generating dispersion. Modulation of the uniform flow then leads to model equations. The coefficients in the model equations are related precisely to the derivatives of the mass flux, momentum flux, and mass density. The theory gives a new perspective from the viewpoint of hydraulics - on how and why key shallow water models like the Korteweg-de Vries equation and Kadomtsev-Petviashvili equations arise in the theory of water waves.

Key words: hydraulics, mass flux, Lagrangian, symmetry, modulation, water waves

\section{Introduction}

Criticality, uniform flows and bulk quantities such as mass flux $(Q)$, total head $(R)$ and the flow force $(S)$ are at the heart of the subject of open-channel hydraulics in one space dimension (Henderson 1966; Abbott 1979). The key properties of uniform flows $\left(h_{0}, u_{0}\right)$, where $h_{0}$ is the constant depth and $u_{0}$ is the horizontal mean velocity, are captured by the mass flux, total head, and momentum flux,

$$
\mathscr{Q}=h_{0} u_{0}, \quad \mathscr{R}=g h_{0}+\frac{1}{2} u_{0}^{2}, \quad \mathscr{S}=\frac{1}{2} h_{0} u_{0}^{2}+\frac{1}{2} g h_{0}^{2},
$$

where $g$ is the gravitational constant, and fluid density is taken to be unity. Here and henceforth $(\mathscr{Q}, \mathscr{R}, \mathscr{S})$ represent $(Q, R, S)$ evaluated on a uniform flow.

There are various ways to define criticality of a uniform flow, but for the purposes of this paper the most useful definition is in terms of the mass flux. The uniform flow is critical if

$$
\left.\mathscr{Q}_{u}\right|_{\mathscr{R} \text { fixed }}=0, \quad \text { where } \quad \mathscr{Q}_{u}:=\frac{\partial \mathscr{Q}}{\partial u_{0}},
$$

(see Figure 1). $\mathscr{R}$ fixed determines $h_{0}$ as a function of $u_{0}$ and so

$$
\left.\mathscr{Q}_{u}\right|_{\mathscr{R} \text { fixed }}=\frac{1}{g}\left(\mathscr{R}-\frac{3}{2} u_{0}^{2}\right)=h_{0}-\frac{u_{0}^{2}}{g},
$$

recovering the usual "Froude number unity condition". A curiosity in the theory of open channel hydraulics is that the second derivative of $\mathscr{Q}$,

$$
\kappa=\left.\mathscr{Q}_{u u}\right|_{\mathscr{R} \text { fixed }},
$$

$\dagger$ Email address for correspondence: T.Bridges@surrey.ac.uk 


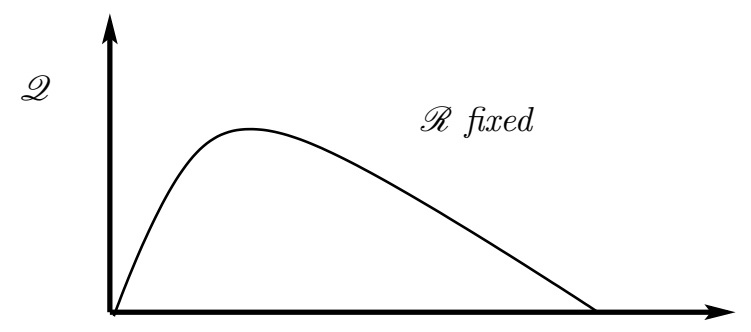

$u_{0}$

FiguRE 1. Mass flux versus uniform velocity for fixed total head

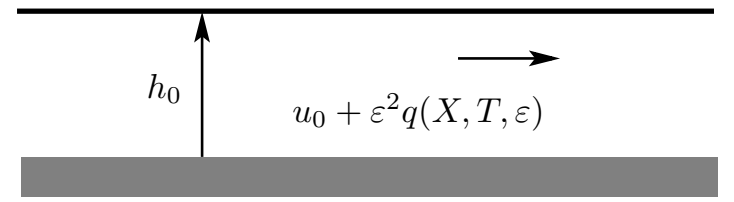

Figure 2. Modulation of the uniform flow

evaluated at criticality is never considered. $\kappa$ is a curvature, and its calculation in this case is elementary: $\kappa=-\frac{3}{g} u_{0}$, and there is no obvious reason it might be interesting.

However, remarkably, $\kappa$ appears as the critical coefficient of the nonlinearity in the $\mathrm{KdV}$ equation when dispersion emerges in shallow water hydrodynamics. To show this, and its generalization to two-dimensions, it is useful to establish how and why the KdV equation emerges from a uniform flow.

The traditional argument for the emergence of dispersion in shallow water, going back to the paper of Korteweg \& de Vries (1895), starts with a quiescent background flow, the shallow-water limit, small amplitude, and the assumption that "amplitude balances dispersion". This latter assumption can be manifested by expanding the governing equation in terms of two parameters (dispersion parameter and amplitude parameter) and taking the limit to zero with their ratio constant. This balancing takes place on a trivial background flow and leads to a two-way Boussinesq equation. Unidirectionalization is then the splitting of the Boussinesq equation into left-running and right-running $\mathrm{KdV}$ equations. See $§ 13.11$ of Whitham (1974) for a discussion of this strategy for deriving $\mathrm{KdV}$. The standard form for the two emergent $\mathrm{KdV}$ equations in dimensional coordinates is

$$
U_{t} \pm \sqrt{g h_{0}} U_{x}+\frac{3}{2} U U_{x} \pm \frac{h_{0}^{2}}{6} \sqrt{g h_{0}} U_{x x x}=0 .
$$

It is derived relative to a moving frame of reference, with the frame speed $c= \pm \sqrt{g h_{0}}$. The \pm signs here are an indication of left and right-running versions.

A new approach to the derivation of the $\mathrm{KdV}$ equation was presented in Bridges (2013) based on modulation of the uniform flow near criticality. This modulation is shown schematically in Figure 2. In the modulation $X=\varepsilon x, T=\varepsilon^{3} t$ and $\varepsilon$ is a measure of the distance from criticality. Carrying out the modulation results in the following equation for $q$,

$$
2 \mathscr{M}_{u} q_{T}+\mathscr{Q}_{u u} q q_{X}+\mathscr{K} q_{X X X}=0,
$$

where $\mathscr{M}$ is the mass density, evaluated on a uniform flow, with $\mathscr{R}$ fixed. If $(x, z)$ coordinates are used with $z$ vertically upward, $z=0$ the horizontal bottom, and the free 
surface at $z=\eta(x, t)$, then the mass conservation law for the full water wave problem is

$$
M_{t}+Q_{x}=0, \quad M=\int_{0}^{\eta} \mathrm{d} z, \quad Q=\int_{0}^{\eta} u(x, z, t) \mathrm{d} z,
$$

with fluid density taken to be unity. For uniform flow, $\mathscr{M}=h_{0}=\frac{1}{g}\left(\mathscr{R}-\frac{1}{2} u_{0}^{2}\right)$ and so $\mathscr{M}_{u}=-\frac{u_{0}}{g}$. Substituting the expressions for $\mathscr{M}_{u}, \mathscr{Q}_{u u}$, and $\mathscr{K}=\frac{h_{0}^{3}}{6}$ into (1.2) and noting that $u_{0}= \pm \sqrt{g h_{0}}$ at criticality reduces it exactly to (1.1), except that (1.2) is relative to a laboratory frame (but Galilean invariance assures that they are equivalent).

The detailed derivation leading from $u=u_{0}+\varepsilon^{2} q$ to the precise form (1.2) is given in Bridges (2013). Substitution of the modulation ansatz into the governing equations, and expansion of all terms in powers of $\varepsilon$, is straightfoward. The key new idea is how to relate the coefficients in the resulting $\mathrm{KdV}$ equation to the components of the conservation law evaluated on the uniform flow. That theory relies on the fact the the governing equations are deduced from a Lagrangian, and the fact that Noether's theorem gives a precise connection in this setting between symmetries and conservation laws. Indeed, since the Lagrangian-symmetry connection is quite general, it can be established that criticality, suitably generalised, is a universal mechanism for the emergence of the KdV equation, and gives a rule for constructing the coefficients (cf. Bridges 2013).

The purpose of this paper is to generalise the emergence of nonlinearity and dispersion via modulation to the case of two-horizontal space dimensions, giving a new theory for the emergence of the Kadomtsev-Petviashvili (KP) equation. Given a uniform flow $\left(h_{0}, u_{0}, v_{0}\right)$ the mass flux is now vector valued,

$$
\mathscr{Q}\left(u_{0}, v_{0}\right)=h_{0} u_{0} \quad \text { and } \mathscr{P}\left(u_{0}, v_{0}\right)=h_{0} v_{0}, \quad \text { with } \quad g h_{0}+\frac{1}{2}\left(u_{0}^{2}+v_{0}^{2}\right)=\mathscr{R},
$$

and the generalisation of (1.2) is the KP equation,

$$
2 \mathscr{M}_{u} q_{T}+\mathscr{Q}_{u u} q q_{X}+\mathscr{K}_{X X X}+\mathscr{P}_{v} p_{Y}=0 \quad \text { and } \quad q_{Y}=p_{X} .
$$

The first three coefficients are the same as the KdV equation in (1.2), and the new coefficient, $\mathscr{P}_{v}$, is determined from the transverse mass flux (it is immediate from (1.4) that $\mathscr{P}_{v}=h_{0}$ at criticality). The mass conservation law in three dimensions is

$$
M_{t}+Q_{x}+P_{y}=0, \quad \text { with } P=\int_{0}^{\eta} v(x, y, t) \mathrm{d} z,
$$

with $\mathscr{P}$ equal to $P$ evaluated on a uniform flow.

The KP equation (1.5) arises due to modulation of the uniform flow near criticality. But what is criticality in this case? The theory shows that the right generalisation is

$$
\left.\mathscr{Q}_{u}\right|_{\mathscr{R} \text { fixed }}=0 \text { and }\left.\quad \mathscr{P}_{u}\right|_{\mathscr{R} \text { fixed }}=0 \text {. }
$$

The latter requirement does not appear in the classic hydraulics literature, but it appears naturally as the required condition in the modulation theory. Evaluation of the coefficients on the uniform flow gives the KP equation that is found in the textbooks (e.g. Dingemans 1997; Johnson 1997; Mei 1989; Osborne 2010).

There are three interesting precedents for the theory here. In Benjamin \& Lighthill (1954) the steady KdV equation is derived based on a perturbation of the uniform flow, with the mass flux $(Q)$, total head (Bernoulli constant) $(R)$ and flow force $(S)$ playing a central role. However, the Benjamin-Lighthill theory is for the steady problem only, and the coefficients in the KdV equation are not expressed in terms of the properties of the uniform flow (for example the coefficient of the nonlinearity is not the curvature of $Q$ ). Moreover, in Benjamin \& Lighthill (1954) it is the extension to finite amplitude waves 
of $S, Q$ and $R$ that is important, whereas here it is $Q$ and $R$ evaluated on the uniform flow that is important.

The second precedent is Whitham modulation theory (WMT) (Whitham 1974). Indeed, the modulation theory here can be considered a generalization of Whitham modulation theory. If the modulation ansatz is changed so that the slow time variable is $T=\varepsilon t$, then WMT is obtained, which is a first order PDE and dispersionless. To get dispersion the slow time scale needs to be changed and degeneracy is necessary.

The third precedent is the classical method of multple scales (e.g. Grimshaw 2005, and references therein). In this approach, an amplitude function $A(X, T)$ which multiplies the eigenfunction of the linear problem is introduced, where $X$ and $T$ are slow space and time variables. A key in this approach is that a solvability condition is central and generates the KdV equation at third order. This combination of multiple scales and a solvability condition is particularly useful for deriving the KdV and KP equation for other settings such as internal waves in stratified flow.

An outline of the paper is as follows. Firstly, in $\S 2$ and $\S 3$, the derivation of KP from modulation of two-dimensional uniform flows in the full water-wave problem is sketched. In $\S 3.1$ it is shown that the new KP equation agrees with the KP equation in textbooks. A curiosity is that the theory works directly with the uniform flows and the dispersion relation is never used. This missing link is surprising since the dispersion relation is important is almost every derivation of model equations in the theory of nonlinear waves. In $\S 4$ it is shown that indeed the dispersion relation is in the theory - it is implicit. Some discussion and concluding remarks are in $\S 5$.

\section{Water waves and multi-dimensional uniform flows}

Consider the inviscid irrotational water-wave problem in three dimensions $(x, y, z, t)$ with $0 \leqslant z \leqslant \eta(x, y, t)$, and velocity potential $\phi(x, y, z, t)$. The governing equations can be deduced from Luke's Lagrangian

$$
\mathscr{L}=\iiint \mathcal{L} \mathrm{d} x \mathrm{~d} y \mathrm{~d} t,
$$

with

$$
\mathcal{L}=\int_{0}^{\eta}\left[\phi_{t}+\frac{1}{2}\left(\phi_{x}^{2}+\phi_{y}^{2}+\phi_{z}^{2}\right)+g z-R\right] \mathrm{d} z,
$$

where $R$ is the Bernoulli function. The Euler-Lagrange equations deduced from this functional produce the governing equation for water waves ( 113.2 of Whitham 1974).

As solutions of the full water wave problem, uniform flows are

$$
\eta(x, y, t)=h_{0} \quad \text { and } \quad \phi(x, y, z, t)=\theta=u_{0} x+v_{0} y+\theta_{0} .
$$

Bernoulli's equation then gives the expression for $\mathscr{R}$ in (1.4). For the time-depdendent problem the Bernoulli "constant" depends on time, although here it is taken to be a constant. (It is an interesting exercise to modulate the Bernoulli function as well, but it leads to the same KdV equation - see comments in $\S 5$.)

The uniform flow is intrinsically connected to the symmetry $a \mapsto \phi+a$; that is, the velocity potential is invariant under addition of an arbitrary constant. Although this symmetry appears to be trivial physically, it generates via Noether's theorem the mass conservation law (Benjamin \& Olver 1982), and it is modulation of the mass conservation law that will result in the KP equation in (1.5). 
2.1. Criticality of multi-dimensional uniform flows

Evaluate the components of the mass conservation law on the basic state $\left(h_{0}, u_{0}, v_{0}\right)$ with $h_{0}$ determined from the Bernoulli equation,

$$
\begin{aligned}
\mathscr{M}\left(u_{0}, v_{0}\right) & =h_{0}=\frac{1}{g}\left(\mathscr{R}-\frac{1}{2} u_{0}^{2}-\frac{1}{2} v_{0}^{2}\right) \\
\mathscr{Q}\left(u_{0}, v_{0}\right) & =h_{0} u_{0}=\frac{u_{0}}{g}\left(\mathscr{R}-\frac{1}{2} u_{0}^{2}-\frac{1}{2} v_{0}^{2}\right) \\
\mathscr{P}\left(u_{0}, v_{0}\right) & =h_{0} v_{0}=\frac{v_{0}}{g}\left(\mathscr{R}-\frac{1}{2} u_{0}^{2}-\frac{1}{2} v_{0}^{2}\right) .
\end{aligned}
$$

The generalisation of $\left.\mathscr{Q}_{u}\right|_{\mathscr{R} \text { fixed }}$ is the matrix of first derivatives

$$
\left[\begin{array}{ll}
\mathscr{Q}_{u} & \mathscr{Q}_{v} \\
\mathscr{P}_{u} & \mathscr{P}_{v}
\end{array}\right]=\frac{1}{g}\left[\begin{array}{cc}
\mathscr{R}-\frac{3}{2} u_{0}^{2}-\frac{1}{2} v_{0}^{2} & -u_{0} v_{0} \\
-u_{0} v_{0} & \mathscr{R}-\frac{3}{2} u_{0}^{2}-\frac{1}{2} v_{0}^{2}
\end{array}\right]=\frac{1}{g}\left[\begin{array}{cc}
g h_{0}-u_{0}^{2} & -u_{0} v_{0} \\
-u_{0} v_{0} & g h_{0}-v_{0}^{2}
\end{array}\right],
$$

after substituting for $\mathscr{R}$.

What is the appropriate generalisation of criticality? Setting the determinant to zero

$$
0=\operatorname{det}\left[\begin{array}{ll}
\mathscr{Q}_{u} & \mathscr{Q}_{v} \\
\mathscr{P}_{u} & \mathscr{P}_{v}
\end{array}\right]=\frac{h_{0}}{g}\left(g h_{0}-\left(u_{0}^{2}+v_{0}^{2}\right)\right),
$$

recovers the usual Froude number unity condition with the two-dimensional velocity field. Here the generalisation of criticality is taken to be

$$
0=\mathscr{Q}_{u}=g h_{0}-u_{0}^{2} \quad \text { and } \quad 0=\mathscr{P}_{u}=-u_{0} v_{0},
$$

which gives a zero determinant but also retains the one-dimensional criticality. The second condition identifies the $x$-direction as dominant. These conditions arise naturally in the modulation theory leading to the KP equation, but are heretofore unknown in the water-wave literature.

\section{Symmetry, modulation and the KP equation}

The dependent variables in the water wave problem are

$$
U(x, y, z, t)=\left(\begin{array}{c}
h(x, y, t) \\
\phi(x, y, z, t)
\end{array}\right)
$$

with basic state,

$$
\widehat{U}\left(\theta, u_{0}, v_{0}\right)=\left(\begin{array}{c}
\frac{1}{g}\left(\mathscr{R}-\frac{1}{2} u_{0}^{2}-\frac{1}{2} v_{0}^{2}\right) \\
\theta
\end{array}\right), \quad \text { with } \quad \theta=u_{0} x+v_{0} y+\theta_{0},
$$

where $\theta_{0}$ is an arbitary constant.

Now introduce the modulation: a solution of the full water-wave problem is proposed in the form

$$
U(x, y, z, t)=\widehat{U}\left(\theta+\varepsilon \psi(X, Y, T, \varepsilon), u_{0}+\varepsilon^{2} q(X, Y, T, \varepsilon), v_{0}\right)+\varepsilon^{3} W(X, Y, T, z, \varepsilon),
$$

with

$$
q=\psi_{X}, \quad X=\varepsilon x, \quad Y=\varepsilon^{2} y, \quad T=\varepsilon^{3} t .
$$

The solution is an ansatz. It is substituted into the governing equations, everything is expanded in powers of $\varepsilon$, and the system is solved order by order, with a solvability condition appearing at each order.

This expansion is to be contrasted with a classical multiple-scales formulation,

$$
U(x, y, z, t)=\widehat{U}\left(\theta, u_{0}, v_{0}\right)+\sum_{j=1}^{\infty} \varepsilon^{j} W_{j}(X, Y, T, z) .
$$


In principle they are the same since the $\widehat{U}$ arguments in (3.1) can be expanded in Taylor series and absorbed in something like the second term on the right-hand side of (3.2). The advantage of (3.1) is that the derivatives of $\widehat{U}$ carry information that feeds into the modulation equation.

Expanding out the terms in (3.1) gives the following expressions for $(\eta, \phi)$,

$$
\begin{aligned}
& \eta=h_{0}-\frac{u_{0}}{g} \varepsilon^{2} q-\frac{1}{2 g} \varepsilon^{4} q^{2}+\varepsilon^{3} H(X, Y, T, \varepsilon) \\
& \phi=\theta+\varepsilon \psi+\varepsilon^{3} f(X, Y, T, z, \varepsilon)
\end{aligned}
$$

$H$ and $f$ are further expanded in powers of $\varepsilon$, up to third order. The expansions are all substituted into the governing equations. The general result for the $2 \mathrm{D}$ case is given in Bridges (2013), and so here just a sketch of the additional theory is given, with the only new result being the appearance of $\mathscr{P}_{v}$ as the new coefficient of transverse dispersion.

The terms proportional to $\varepsilon^{0}$ and $\varepsilon^{1}$ just recover the equations for the uniform flow, and the second order equation gives the homogeneous linearisation about the uniform flow.

At third order the solvability condition requires $\mathscr{Q}_{u}=0$, and gives the leading order terms for $H, f$,

$$
\begin{aligned}
H_{1} & =-\frac{v_{0}}{g} \psi_{Y} \\
f_{1} & =-\frac{1}{2} h_{0}^{2} z^{2} q_{X}+\alpha,
\end{aligned}
$$

where $\alpha$ is an arbitrary function of $X, Y, T$.

At fourth order, solvability requires $\mathscr{P}_{v}=0$. Establishing this follows the same strategy (relating components of conservation laws via symmetry to the uniform flows) as for $\mathscr{Q}_{u}=0$ in Bridges (2013). The fourth order solution is

$$
\begin{aligned}
H_{2} & =-\frac{1}{g} \psi_{T}+\frac{u_{0} h_{0}^{2}}{2 g} q_{X X}-\frac{u_{0}}{g} \alpha_{X} \\
f_{2} & =\beta,
\end{aligned}
$$

where $\beta$ is an arbitrary function of $X, Y, T$.

At fifth order a linear system of the following form is found

$$
\mathbf{L} W_{3}=() q_{T}+() q_{X X X}+() q q_{X}+() \psi_{Y Y} .
$$

The coefficients in the first three terms are given in Bridges (2013), with the coefficient of $\psi_{Y Y}$ being new. Application of the solvability condition then gives

$$
a_{1} q_{T}+a_{2} q q_{X}+a_{3} q_{X X X}+a_{4} \psi_{Y Y}=0 .
$$

These coefficients are complicated expressions in terms of inner products. The key next step is to use the connection between symmetry and conservation laws to give simple expressions for the coefficients. In Bridges (2013) it is shown that $a_{1}=-2 \mathscr{M}_{u}, a_{2}=$ $-\mathscr{Q}_{u u}$, and $a_{3}=-\mathscr{K}$. A similar argument shows that $a_{4}=-\mathscr{P}_{v}$. Defining $p=\psi_{Y}$ gives $p_{X}=q_{Y}$ and then equation (3.4) becomes

$$
2 \mathscr{M}_{u} q_{T}+\mathscr{Q}_{u u} q q_{X}+\mathscr{K} q_{X X X}+\mathscr{P}_{v} p_{Y}=0,
$$

completing the derivation of (1.5). The case where the dispersions have the same sign, $\mathscr{K} \mathscr{P}_{v}>0$, is KP-II and is the case associated with shallow water when gravity is dominant. The case $\mathscr{K} \mathscr{P}_{v}<0$ is KP-I and is a model in shallow water when surface tension is dominant. Note that when surface tension is present, the only effect is on the coefficient $\mathscr{K}$. Surface tension can not affect the other coefficients since they are determined by the uniform flow! 


\subsection{Comparison with the standard form of $K P$}

Evaluating the coefficients in KP (1.5) on the uniform flow at criticality and using the expressions for $\mathscr{K}$ and $\mathscr{P}_{v}$ from (4.2), it becomes

$$
\left(-2 \frac{u_{0}}{g} q_{T}-3 \frac{u_{0}}{g} q q_{X}+\frac{h_{0}^{3}}{3} q_{X X X}\right)_{X}+h_{0} q_{Y Y}=0,
$$

or, after dividing through by $-2 u_{0} / g$ and imposing criticality, $u_{0}=\mp \sqrt{g h_{0}}$,

$$
\left(q_{T}+\frac{3}{2} q q_{X} \pm \sqrt{g h_{0}} \frac{h_{0}^{2}}{6} q_{X X X}\right)_{X} \pm \frac{1}{2} \sqrt{g h_{0}} q_{Y Y}=0 .
$$

The standard form for the two emergent KP equations in dimensional coordinates is

$$
\frac{\partial}{\partial x}\left( \pm \eta_{t}+\sqrt{g h_{0}} \eta_{x}+\frac{1}{2} \sqrt{\frac{g}{h_{0}}} \eta \eta_{x}+\frac{h_{0}^{2}}{6} \sqrt{g h_{0}} \eta_{x x x}\right)+\frac{1}{2} \sqrt{g h_{0}} \eta_{y y}=0 .
$$

This is the "height form" of the KP equation. It is derived relative to a moving frame of reference, with the frame speed $c= \pm \sqrt{g h_{0}}$, and the background velocity field is quiescent. The \pm signs here are an indication of left and right-running versions. A derivation of this equation can be found in $\S 6.8$ of Dingemans (1997) (see equation (6.275c) on page 776). The "velocity form" of KP is obtained by substituting $\eta=3 \sqrt{\frac{h_{0}}{g}} u$, giving

$$
\frac{\partial}{\partial x}\left(u_{t} \pm \sqrt{g h_{0}} u_{x}+\frac{3}{2} u u_{x} \pm \frac{h_{0}^{2}}{6} \sqrt{g h_{0}} u_{x x x}\right) \pm \frac{1}{2} \sqrt{g h_{0}} u_{y y}=0 .
$$

It is this equation which corresponds to (3.6).

\section{Implicit role of the dispersion relation}

The dispersion relation, associated with the linearisation about the uniform flow, does not play an explicit role in the modulation theory for emergence of the KP equation. It is implicit. Replacing $\partial_{T}$ by $-\mathrm{i} \omega, \partial_{X}$ by $\mathrm{i} k$, and $\partial_{Y}$ by $\mathrm{i} \ell$, the linear part of (1.5) becomes

$$
-2 \mathscr{M}_{u} \mathrm{i} \omega+\mathrm{i} k \mathscr{Q}_{u}+\mathscr{K}(\mathrm{i} k)^{3}+\mathscr{P}_{v} \mathrm{i} \frac{\ell^{2}}{k}=0 .
$$

This is just the leading order expansion of the exact dispersion relation

$$
\left(\omega-k u_{0}-\ell v_{0}\right)^{2}=g \kappa \tanh \left(\kappa h_{0}\right), \quad \kappa=\sqrt{k^{2}+\ell^{2}} .
$$

A calculation shows that

$$
\omega_{k}=0 \quad \Leftrightarrow \quad \mathscr{Q}_{u}=0 \quad \text { and } \quad \omega_{k \ell}=0 \quad \Leftrightarrow \quad \mathscr{P}_{u}=0 .
$$

This correspondence can be verified by direct calculation, but in fact can be proved directly from the governing equations, but is outside the scope of this paper. Carrying the calculation to higher order shows that

$$
\begin{aligned}
2 \mathscr{M}_{u} \frac{1}{6} \omega_{k k k} & =\delta \frac{h_{0}^{3}}{3}=\delta \mathscr{K} \\
k \mathscr{M}_{u} \omega_{\ell \ell} & =\delta h_{0}=\delta \mathscr{P}_{v} .
\end{aligned}
$$

where $\delta=\left( \pm \frac{u_{0}}{\sqrt{g h_{0}}}\right)$. Since $u_{0}= \pm \sqrt{g h_{0}}, \delta$ can be taken to be unity. All derivatives are evaluated at $k=\ell=0$ except $\omega_{\ell \ell}$ which is evaluated at $\ell=0$ only. One by-product is that the coefficients of the classical dispersion relation for irrotational water waves (4.1) can be given an interpretation in terms of the geometry of uniform flows. 


\section{Concluding remarks}

The main result in this paper is that the KP equation, the same KP equation that appears in textbooks, arises due to a much simpler mechanism than previously thought, and that mechanism is closely tied to classical hydraulics.

Since the mechanism involves starting with a Lagrangian with symmetry, and looking for degeneracy of the flux vector of the attendant conservation law, the theory should generalise to many other situations. For example, in Bridges (2013), it is shown how the $\mathrm{KdV}$ equation can be found in many other situations. That theory generalises to KP.

Anywhere criticality appears in hydrodynamics, one can expect a generalisation of the theory here to be developable. For example, the theory of criticality for two layer fluids (e.g. Bridges \& Donaldson 2007) is manifestly more interesting, and the theory in this paper can be suitably generalised to generate a theory for the emergence of dispersion in that setting by modulating the two-layer uniform flow. Another example is finiteamplitude criticality of Stokes waves (Bridges \& Donaldson 2006).

Changing the scaling will change the modulation equation. For example, modulation of the uniform flow with the ansatz (3.1) but with the scaling $X=\varepsilon x$ and $T=\varepsilon^{2} t$ will generate a two-way Boussinesq equation, with coefficients determined by derivatives of the uniform flow.

The basic state (2.2) can be include time modulation, replacing $\theta$ with

$$
\theta=u_{0} x+v_{0} y-r t+\theta_{0} .
$$

and replacing the modulation (3.1) with

$$
U(x, y, z, t)=\widehat{U}\left(\theta+\varepsilon \psi, u_{0}+\varepsilon^{2} q, v_{0}, r+\varepsilon^{4} R\right)+\varepsilon^{3} W(X, Y, T, z, \varepsilon),
$$

with $R=-\psi_{T}$, and $\varepsilon^{4}$ chosen so that $q_{T}+R_{X}=0$ is in balance. However, at fifth order the same KP equation emerges. The full details of this derivation (and other possible modulations like $v_{0} \mapsto v_{0}+\varepsilon^{3} V$ ) will be given elsewhere.

A moving frame can be included by introducing the transformation $x \mapsto x-c t$, where $c$ is the frame speed. Examples in the $1+1$ setting are discussed in Bridges (2013).

\section{REFERENCES}

Аввотт, M. B. 1979 Computational Hydraulics. London: Pitman Publishers.

Benjamin, T. B. \& Lighthill, M. J. 1954 On cnoidal waves and bores. Proc. Roy. Soc. Lond. A 224, 448-460.

Benjamin, T. B. \& Olver, P. J. 1982 Hamiltonian structure, symmetries and conservation laws for water waves. J. Fluid Mech. 125, 137-185.

BRIDGES, T. J. 2013 A universal form for the emergence of the Korteweg-de Vries equation. Proc. Roy. Soc. Lond. A 469, 20120707.

Bridges, T. J. \& Donaldson, N. M. 2006 Secondary criticality of water waves. Part 1: Definition, bifurcation and solitary waves. J. Fluid Mech. 565, 381-417.

Bridges, T. J. \& Donaldson, N. M. 2007 Reappraisal of criticality for two-layer flows and its role in the generation of internal solitary waves. Phys. Fluids 19, 072111.

Dingemans, M. W. 1997 Wave Propagation over Uneven Bottoms. Part 2: Nonlinear Wave Propagation. Singapore: World Scientific Ltd.

Grimshaw, R. H. J. 2005 Korteweg-de Vries equation. In Nonlinear Waves in Fluids: Recent Advances and Modern Applications (ed. R. H. J. Grimshaw), pp. 1-28. Springer-Verlag.

Henderson, F. M. 1966 Open Channel Flow. London: MacMillan Publishers.

Johnson, R. S. 1997 A Modern Introduction to the Mathematical Theory of Water Waves. Cambridge: Cambridge University Press.

KorteweG, D. J. \& DE VRIES, G. 1895 On the change of form of long waves advancing in a rectangular canal, and on a new type of long stationary waves. Phil. Mag. 39, 422-443. 
Mei, C. C. 1989 The Applied Dynamics of Ocean Surface Waves. Singapore: World Scientific. Osborne, A. R. 2010 Nonlinear Ocean Waves and the Inverse Scattering Transform. London: Academic Press, Elsevier.

Whitham, G. B. 1974 Linear and Nonlinear Waves. New York: John Wiley \& Sons. 